\title{
Personalie
}

\section{Gerhard Knorz †}

\section{Prof. Dr. Gerhard Knorz, geboren am 21. Juni 1951, ist am 16. März 2015 im Alter von 63 Jahren verstorben.}

DOI 10.1515/iwp-2015-0036

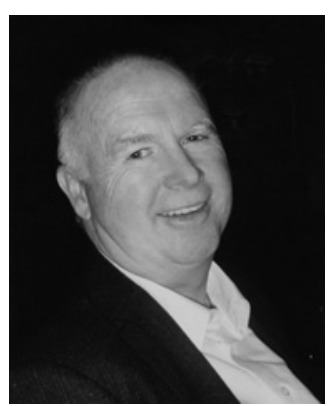

Seine informationswissenschaftliche Laufbahn begann 1978 an der TU Darmstadt, wo er nach dem Diplom in Informatik unter der Leitung von Prof. Dr. Gerhard Lustig als Wissenschaftlicher Mitarbeiter Lehrveranstaltungen u.a. zur Automatischen Indexierung und Information Retrieval sowie zur Linguistischen Datenverarbeitung durchführte und an mehreren Projekten zur automatischen Verschlagwortung arbeitete: Wörterbuchentwicklung für die automatische Indexierung (1987 bis 81), Automatische Indexierung und Retrieval (1981 bis 84) und AIR/PHYS, einer Pilotanwendung von automatischen Indexing- und Retrievalverfahren mit der Datenbank PHYS des Fachinformationszentrums Karlsruhe (1984 bis 86). All dies führte in eine ab 1985 laufende praktische Anwendung für eine große Datenbasis beim FIZ Karlsruhe. Die geglückte Verbindung von Wissenschaft und Praxis war vorbildhaft für die weitere Entwicklung. Wichtigster Beitrag von Gerhard Knorz war die Anwendung eines höchst effizienten Verfahrens aus der Mustererkennung (Polynomklassifikatoren) für die Zwecke der automatischen Indexierung, womit er die spätere Verwendung von Ansätzen aus dem Bereich des maschinellen Lernens für die Dokumentklassifikation vorwegnahm. Diese Arbeiten bilden den Kern seiner 1983 fertig gestellter Dissertation „Automatisches Indexieren als Erkennen abstrakter Objekte“, für die er mit dem Erich-Pietsch-Preis der DGI (damals noch DGD) ausgezeichnet wurde.

Im Anschluss an die genannten Projekte leitete Gerhard Knorz dann zusammen mit Brigitte Endres-Niggemeier ein Projekt zur wissensbasierten automatischen Erfassung bibliografischer Daten am Beispiel von Kernzeitschriften des Faches Physik. Später verschob sich sein Forschungsinteresse mehr hin zu Ontologien.

Im Juli 1983 erhielt Gerhard Knorz eine Professur an der Hochschule (damals noch Fachhochschule) Darm- stadt. Nach der Gründung des Fachbereichs Information und Dokumentation (IuD) im WS 1985/86 wurde er dort auf eine Professur für das Fach Dokumentationssprachen berufen. Hier beteiligte er sich fortan maßgeblich an der Entwicklung der informationswissenschaftlichen Studiengänge, zunächst von 1992 bis 1993 als stellvertretender Dekan, später von 1993 bis 1995 und von 1999 bis 2001 als Dekan des Fachbereichs Information und Dokumentation und als Vorsitzender des Lehr- und Studienausschusses des Studiengangs Informations- und Wissensmanagement. Außerdem nahm er als Mitglied im Konvent und im Rat der Fachhochschule und in der Ratskommission Forschung und Entwicklung Funktionen in der Selbstverwaltung auf zentraler Ebene wahr.

Von 2002 bis 2010 engagierte er sich dort als Vizepräsident für Informations- und Qualitätsmanagement und war dabei u. a. für die Einführung der Online-Bewerbung, die Modernisierung und Neuorganisation der Informationstechnik der gesamten Hochschule sowie die Einführung eines konsistenten Corporate Designs der Hochschule zuständig - und er führte das jährliche Campusfest ein.

In zwei Forschungssemestern setzte sich Gerhard Knorz mit zeitgemäßen Lehrmethoden auseinander, im Sommersemester 1996 mit der Thematik „Neue Formen von Lehre - Multimedia und Netze“ und im Wintersemester 2001/02 mit der Thematik „Informationswissenschaftliche Module für die multimediale netzbasierte Hochschullehre“. Dieses führte zu einem Verbundprojekt von vier deutschen Hochschulen mit dem Ziel E-Learning in den Ingenieur- und Informationswissenschaften $\mathrm{zu}$ fördern. Gerhard Knorz entwickelte die ersten vollständig als Online-Lehrveranstaltungen der Hochschule Darmstadt verfügbaren und als Blended Learning angebotenen Lehrveranstaltungen und bot sie seit 2002 kontinuierlich und mit Erfolg hinsichtlich Nachfrage und Lernergebnis an.

Seit 1996 bis zu seinem Wechsel in die Hochschulleitung (2002) hat Gerhard Knorz sämtliche berufliche Tätigkeiten bis hin zur Beschreibung jedes einzelnen Lehrveranstaltungstermins mit allen verwendeten Unterlagen vollständig im World Wide Web dokumentiert 
(WebSite Methodik). Dieser Informationsservice macht Hochschullehre vollkommen transparent und wurde aus dem gesamten deutschsprachigen Raum mit größenordnungsmäßig 10.000 Seitenzugriffen pro Semestermonat frequentiert. Die Lehrmaterialien betreffen vielfach methodische Aspekte und wurden und werden vermutlich immer noch an vielen Hochschulen verwendet.

Regelmäßig evaluierte Gerhard Knorz seine Lehrveranstaltungen insgesamt und in Teilen. Dass die Ergebnisse dieser Evaluierung (unabhängig von ihrem Ergebnis) auf „WebSite Methodik“ publiziert wurden, betrachteten Lehrende auch anderer Hochschulen als aufschlussreiche Informationsquelle im Hinblick auf die Akzeptanz neuer Lehrformen.

Aus dem Kreis der Darmstädter Absolventinnen und Absolventen kamen auf die Todesnachricht über dreißig Rückmeldungen, stellvertretend sei eine wiedergegeben „Ich habe Herrn Prof. Dr. Knorz sehr geschätzt! Wenn er nicht gewesen wäre, hätte ich vielleicht das Studium an der $\mathrm{FH}$ abgebrochen ... $\mathrm{Zu}$ recht wurde er auch Dekan, denn er hat es wirklich verstanden, die Interessen der Studienfächer mit Interessen der Studenten und Angestellten gleichermaßen zu berücksichtigen, um so immer wieder Lösungswege zu finden ...“

Daneben war Gerhard Knorz auch in wissenschaftlichen Gesellschaften aktiv: In der Gesellschaft für Linguistische Datenverarbeitung (GLDV, heute GSCL) fungierte er von 1985 bis 2000 als Herausgeber des LDVForums (heute JLCL). Die Gründung der Fachgruppe „Information Retrieval“" innerhalb der GI im Jahre 1991 geht maßgeblich auf sein Wirken zurück. Im Hochschulverband Informationswissenschaft war er Mitglied des Vorstands bis 2004 und Beirat der wissenschaftlichen Schriftenreihe. Im Jahr 2000 organisierte Gerhard Knorz das 7. Internationale Symposium für Informationswissenschaft (ISI) in Darmstadt-Dieburg, führte neue Konferenzformate, insbesondere für die Studierenden, ein und erreichte eine Teilnehmerzahl der ISI, die seitdem nie mehr überboten werden konnte. Die Etablierung des sog. Gerhard-Lustig-Preises für die beste informationswissen- schaftliche Abschlussarbeit war seine Idee, die er mit höchstem Engagement in die Realität umsetzte. 2011 nahm Gerhard Knorz - schon an den Rollstuhl gefesselt und auf die Hilfe seiner Frau Gabi angewiesen - zum letzten Mal an einer ISI in Hildesheim teil - und zwar nicht nur als Zaungast, sondern mit vollem Einsatz und vielen Wortbeiträgen bei Panels und Vorträgen.

Gerhard Knorz verfügte über eine scheinbar unerschöpfliche Energie, mit der er Kollegen und Studierende gleichermaßen für seine Ideen begeistern konnte. Er war immer zur Übernahme zusätzlicher Aufgaben bereit, wobei er aber seine übrigen Pflichten nie vernachlässigte. Umso schwerer muss es ihn getroffen haben, als 2008 bei ihm die Krankheit ALS diagnostiziert wurde, die schrittweise seine motorischen Fähigkeiten einschränkte. „Seinen Lebenswillen, seine Lebensfreude, seinen wachen Geist, seine Kreativität, seine Empathie konnte sie ihm nicht nehmen“, so steht es in der Todesanzeige der Familie. Gerd Knorz hielt noch vom Rollstuhl aus OnlineVorlesungen für seine Studierenden. In seinem letzten Lebensjahr versagte auch seine Stimme. Mit Hilfe einer Software zur Erfassung der Blickbewegungen konnte er Texte in den Computer eingeben und E-Mails schreiben, ein Text-zu-Sprache-System mit seiner zuvor aufgenommenen Stimme erlaubte ihm sogar die sprachliche Kommunikation. Seine Begeisterung und Kreativität zur Nutzung innovativer IT-Verfahren hat ihn bis an sein Ende begleitet.

Die Fachwelt verliert mit Gerd Knorz viel zu früh einen wegweisenden Informationswissenschaftler und hochgeschätzten Kollegen und für viele von uns einen Freund, dessen Herzlichkeit und Esprit wir vermissen werden.

Im Namen der Kolleginnen und Kollegen aus Informatik und Informationswissenschaft

Norbert Fuhr, Rainer Kuhlen, Marlies Ockenfeld, Christa Womser-Hacker 